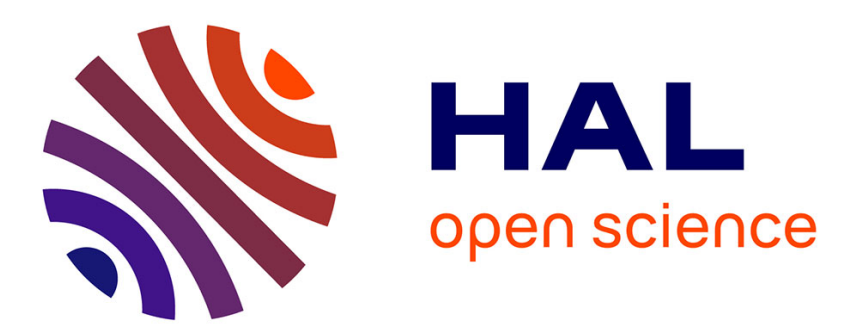

\title{
Removing the CFL stability criterion of the explicit time-domain very high degree spectral-element method with eigenvalue perturbation
}

\author{
Chao Lyu, Yann Capdeville, Gang Lu, Liang Zhao
}

\section{To cite this version:}

Chao Lyu, Yann Capdeville, Gang Lu, Liang Zhao. Removing the CFL stability criterion of the explicit time-domain very high degree spectral-element method with eigenvalue perturbation. Geophysics, 2021, 86 (3), 10.1190/geo2020-0623.1 . hal-03306824

\section{HAL Id: hal-03306824 \\ https://hal.science/hal-03306824}

Submitted on 29 Jul 2021

HAL is a multi-disciplinary open access archive for the deposit and dissemination of scientific research documents, whether they are published or not. The documents may come from teaching and research institutions in France or abroad, or from public or private research centers.
L'archive ouverte pluridisciplinaire $\mathbf{H A L}$, est destinée au dépôt et à la diffusion de documents scientifiques de niveau recherche, publiés ou non, émanant des établissements d'enseignement et de recherche français ou étrangers, des laboratoires publics ou privés. 


\section{GEOPHYSICS $^{\odot}$}

\section{Removing the CFL stability criterion of the explicit time- domain very high degree spectral-element method with eigenvalue perturbation}

\begin{tabular}{|r|l|}
\hline Journal: & Geophysics \\
\hline Manuscript ID & GEO-2020-0623.R2 \\
\hline Manuscript Type: & Technical Paper \\
\hline Keywords: & 2D, acoustic, finite element, wave propagation \\
\hline Manuscript Focus Area: & Seismic Inversion, Seismic Modeling and Wave Propagation \\
\hline \multicolumn{2}{|l}{ Note: The following files were submitted by the author for peer review, but cannot be converted to PDF. } \\
You must view these files (e.g. movies) online. \\
\hline table.tex
\end{tabular}

\section{SCHOLARONE \\ Manuscripts}




\title{
Removing the CFL stability criterion of the explicit time-domain very high degree spectral-element method with eigenvalue perturbation
}

\author{
Chao Lyu ${ }^{1,2}$, Yann Capdeville ${ }^{2}$, Gang Lu ${ }^{1}$, Liang Zhao ${ }^{1 *}$ \\ right-running head: The CFL's removal of SEM \\ ${ }^{1}$ Key Laboratory of Earth and Planetary Physics, Institute of Geology and Geophysics, Chinese \\ Academy of Sciences, Beijing 100029, China. E-mail: zhaoliang@mail.iggcas.ac.cn \\ ${ }^{2}$ Laboratoire de Planétologie et Géodynamique, CNRS, Université de Nantes, UMR-6112, 44322 \\ Nantes, France. E-mail: yann.capdeville@univ-nantes.fr
}

\begin{abstract}
The explicit time-domain spectral-element method (SEM) for synthesizing seismograms has gained tremendous credibility within the seismological community at all scales. Although the recent introduction of non-periodic homogenization has addressed the spatial meshing difficulty of the mechanical discontinuities, the Courant-Friedrichs-Lewy (CFL) stability criterion strictly constrains the maximum time step, which still puts a great burden on the numerical simulation. In the explicit time-domain SEM, the source of instability of using a time step beyond the stability criterion is that some unstable eigenvalues of the updated matrix are larger than what can be accurately simulated. We succeed in removing the CFL stability condition in the explicit time-domain SEM by combining the forward time dispersion-transform method, the eigenvalue perturbation, and the inverse time dispersion-transform method. Our theoretical analyses and numerical experiments both in the homogeneous, moderate and strong heterogeneous models, show that this combination can accurately simulate waveforms with time steps dozens of the CFL limit even towards the Nyquist limit especially for the efficient very high-degree SEM, which abundantly saves the iteration times without suffering from the timedispersion error. It demonstrates a potential application prospect in some situations such as the full waveform inversion that requires multiple numerical simulations for the same model.
\end{abstract}




\section{INTRODUCTION}

The forward simulation of the acoustic and elastic wave equation is a basic and critical part for imaging at all scales from the exploration geophysics to the global Earth scale. In the past two decades, the explicit time-domain spectral-element method (SEM) (Komatitsch and Vilotte, 1998; Chaljub et al., 2007) has been widely used, especially in the academia. The adjoint methods by the two-way SEM modeling have made significant progress in recent regional and global seismic tomographies (Tromp et al., 2005; Fichtner et al., 2009; Zhu et al., 2012; French and Romanowicz, 2015; Fichtner et al., 2018; Capdeville and Métivier, 2018; Tromp, 2020; Lyu et al., 2021). However, the popular explicit time-domain SEM still suffers from two main constraints. On the one hand, each material interface has to be explicitly meshed to ensure the accuracy of the model definition and the precision of the simulation. On the other hand, only a very small time step $\Delta t$ can be used to fulfill the CFL stability condition, because the Gauss-Lobatto-Legendre (GLL) points cluster toward the edges of the elements especially for the very high degree SEM and the model with fine structures. The main intent of the CFL stability condition is governed by the minimum ratio between the size of the grid spacing and the P-wave velocity as follows:

$$
\Delta t \leq C \frac{\Delta x_{\min }}{V_{\mathrm{p}}}
$$

where $C$ is the courant constant, $\Delta x_{\min }$ is the minimum distance between any two GLL points, $V_{\mathrm{p}}$ is the P-wave velocity. In this article, we use the $\Delta t_{\mathrm{CFL}}$ as the maximum stable time step of the secondorder Newmark scheme (Newmark, 1959). And please note that the selection of the available $\Delta t$ close to the $\Delta t_{\mathrm{CFL}}$ of an explicit time-domain SEM in the heterogeneous model has been actually discussed under the control of the CFL stability condition by the local element stability criteria (Cottereau and Sevilla, 2018).

The errors of numerical wave simulation come from the spatial- and time-dispersion errors, and these two kinds of dispersion errors have been proven not relevant (Stork, 2013; Koene et al., 2018; Lyu et al., 2020). That means they accumulate independently during the numerical simulation. Many studies have been focused on how to achieve both high efficiency and accuracy with coarser grids and larger time steps in the forward numerical simulation. The recent introduction of the non- 
periodic homogenization has solved the meshing difficulty by replacing the complex models with their equivalent smooth versions (Capdeville et al., 2010; Cupillard and Capdeville, 2018). The accuracy of the simulated waveforms can be ensured to some degree, which makes the meshing by SEM unrestricted by physical discontinuities anymore. This means that the SEM can flexibly reduce the spatial-dispersion error by tuning the number of elements and the internal polynomial degree $N$ in each tensorial direction for each element. Lyu et al. (2020) validates the high efficiency of using the very high degree SEM $(N=12 \sim 24)$ both for the required memory and the computation time, which makes SEM with very high degree attractive and competitive for solving the wave equation.

Except for the spatial-dispersion error, the selection of the time step is very critical for the simulated accuracy and computational efficiency as well. And please note that the time-dispersion error is only a function of frequency, time step, and propagation time, independent of the propagation path and the complex medium responses (Stork, 2013; Koene et al., 2018). For a model with sufficiently accurate spatial meshing, a converged waveform without spatial and time-dispersion errors can be obtained by using an extremely small $\Delta t$ but with low efficiency. While with the high computational efficiency, the time-dispersion error of using a relatively large time step $\Delta t \leq \Delta t_{\mathrm{CFL}}$ would also contaminate the numerical waveforms for the finite difference (FD) method (Gao et al., 2016) and as well as SEM especially with $N=4$ (Lyu et al., 2020) and even worse for long duration simulations owing to its accumulation over time. Stork (2013) demonstrate that the time-dispersion error can be removed by time-varying filters and interpolation after the wave modelling. Based on this idea, Wang and $\mathrm{Xu}$ (2015) propose that the forward time dispersion transform (FTDT) can predict the time-dispersion error, and the inverse time dispersion transform (ITDT) can eliminate the time-dispersion error from synthetic traces. Koene et al. (2018) mentions that the obtained traces should not only be filtered by the ITDT method as proposed by Wang and Xu (2015), but also the source time function needs to be filtered by the FTDT method before the simulation, which has been validated in the numerical methods including FD, pseudo-spectral (PS) method and SEM. Their combination have been used to obtain better image by the reverse-time migration in real application. All of them introduce a great improvement in the simulated accuracy and computational efficiency with a time step $\Delta t \leq \Delta t_{\mathrm{CFL}}$ by the combination of the FTDT and ITDT methods.

Even so, their adopted time steps are still limited by the CFL stability condition. The spatial 
filtering method (Ecer et al., 2000; Sarris, 2011; Chang and Sarris, 2011; Gao et al., 2019) and the eigenvalue-type method (He et al., 2012; Gaffar and Jiao, 2014; Li, 2014; Li et al., 2014; Jin and Jiao, 2016; Gao et al., 2018) are the two available methods to extend or remove the CFL stability limit of explicit scheme at present.

For the spatial filtering method, in fluid dynamics, Ecer et al. (2000) find that a time step $\Delta t>$ $\Delta t_{\text {CFL }}$ would introduce unstable high-wavenumber wavefields. Therefore, the larger time steps of an explicit scheme could be used if one can filter out the unstable high-wavenumber wavefields. When numerically solving Maxwell's equations by a time step beyond the CFL condition, Sarris (2011) adapt a low-pass filter to remove the unstable high-wavenumbers components. In the seismic field, Gao et al. (2019) extend the stability limit of explicit scheme with spatial filtering for solving the wave equations, and the FTDT and ITDT methods are then used to reduce the large time-dispersion error when using a time step larger than the $\Delta t_{\mathrm{CFL}}$.

For the eigenvalue-type method, in the electromagnetic wave numerical simulation field, $\mathrm{He}$ et al. (2012) propose an explicit time-domain finite-element method (FEM) with unconditional stability, and this method eliminates the dependence of the time step size on the spatial interval. Gaffar and Jiao (2014) address that the source of instability in an explicit time-domain FD using a time step $\Delta t>\Delta t_{\mathrm{CFL}}$ is that the unstable eigenmodes whose eigenvalues are larger than what can be accurately simulated. Jin and Jiao (2016) propose that these unstable modes can be discarded before performing an explicit time marching by using partial eigenvalues solution of the global system matrix. As an alternative, $\mathrm{Li}$ (2014) and Li et al. (2014) adopt the so-called eigenvalue perturbation method to use a time step $\Delta t>\Delta t_{\mathrm{CFL}}$, which normalizes the unstable eigenvalues to one to extend the CFL stability limit. In the seismic field, Etgen (1994) indicates if the singular values are not less than one, especially if they are larger than one, the numerical scheme will develop unstable behavior. Gao et al. (2018) also illustrates that for a given time step $\Delta t \leq \Delta t_{\mathrm{CFL}}$, the eigenvalues of the updated matrix would be distributed along the unit circle; However, for a time step $\Delta t>\Delta t_{\mathrm{CFL}}$, some eigenvalues would be distributed outside of the unit circle. This is the reason of unstable phenomena of the acoustic wave numerical simulation. Gao et al. (2018) indicate that combining the FTDT method, the eigenvalue perturbation and the ITDT method can simulate highly accurate waveforms when applying a time step several times of the $\Delta t_{\mathrm{CFL}}$ in the FD framework of solving 
wave equation.

To our knowledge, the combination of FTDT, the eigenvalue perturbation and ITDT methods has received a lot of attention in the FD framework, but the related research in the SEM framework is rarely studied. No method has been proposed to overcome the CFL stability limit for the explicit time-domain SEM in the acoustic wave simulation from previously published literature.

In this paper, following the validity of the combination of the FTDT and ITDT methods to reduce the time-dispersion error in the SEM framework in Koene et al. (2018), and the successful integration of the FTDT and ITDT methods with eigenvalue perturbation in the FD framework in Gao et al. (2018), we combine these three methods to remove the CFL limit in the explicit timedomain SEM simulation of solving the acoustic wave equation. We first illustrate the theoretical analyses of the combined methods in the SEM framework with the detailed introductions of the eigenvalue perturbation, FTDT and ITDT methods. Then, the associated numerical experiments are performed in the $2 \mathrm{D}$ homogeneous and heterogeneous cases by the SEM with time steps $\Delta t>$ $\Delta t_{\mathrm{CFL}}$

\section{METHODOLOGY}

In this section, we first briefly introduce the numerical simulation of 2D acoustic wave equation by the SEM, then illustrate how the CFL stability limit is removed by the eigenvalue perturbation and finally clarify how the resultant time-dispersion error yielded by $\Delta t>\Delta t_{\mathrm{CFL}}$ is eliminated by the FTDT and ITDT methods.

\section{D acoustic wave equation}

The acoustic waves propagating is governed by the equation of motion

$$
\left\{\begin{array}{l}
\frac{1}{\kappa} \ddot{q}=\nabla \cdot \dot{\mathbf{u}}+f \\
\dot{\mathbf{u}}=\frac{1}{\rho} \nabla q,
\end{array}\right.
$$


where $q$ is the velocity potential, $\kappa(\mathbf{x})$ is the acoustic bulk modulus, $\rho(\mathbf{x})$ is the (mass) density, $\mathbf{u}(\mathbf{x}, t)$ is the displacement vector, and $f(\mathbf{x}, t)$ is a scalar source term. Generally, the acoustic medium is fully described by only two parameters, namely, the density $\rho(\mathbf{x})$ and the sound speed $V(\mathbf{x})$ so that $\kappa(\mathbf{x})=\rho(\mathbf{x}) V^{2}(\mathbf{x})$, and the density is kept constant during the numerical experiments below. And the boundary conditions are free normal stress all around the domain, and the initial velocity potential $q$ and its first time derivative $\dot{q}$ are zero.

\section{Removing the CFL limit of the explicit time-domain SEM}

Like in a standard finite-element method, for generating spatial meshing discretization, the original domain is discretized into nonoverlapping quadrilateral elements. Then the ordinary differential equation in SEM could be built by assembling the element mass and stiffness matrices, which is based on the numerical integration of the weak form of the equation 2 (Komatitsch and Vilotte, 1998). The resultant matrix expression of the acoustic wave equation 2 by the SEM can be written as:

$$
M \frac{\partial^{2} Q}{\partial t^{2}}+K Q=F
$$

where $M$ is the diagonal global mass matrix, $K$ is the sparse global stiffness matrix, $F$ is the source vector, $Q$ and $\frac{\partial^{2} Q}{\partial t^{2}}$ are the unknown velocity potential and its acceleration vector.

And then, for the temporal discretization, we only consider the second-order accurate temporal finite-difference operator, which is the most commonly used time-marching scheme in the SEM (Komatitsch and Vilotte, 1998; Chaljub et al., 2007). The form of equation 3 is changed as follows:

$$
Q^{n+1}-2 Q^{n}+Q^{n-1}=\underbrace{-\Delta t^{2} M^{-1} K}_{A} Q^{n}+\Delta t^{2} M^{-1} F^{n}
$$

where $n$ is related to the time moment $n \Delta t$. Taking a structural meshing as an example, the size of the matrix $A=-\Delta t^{2} M^{-1} K$ is $\left(N_{x} \times N_{z}\right)^{2}, N_{x}$ and $N_{z}$ are the number of grid points of SEM along the $\mathrm{x}$ and $\mathrm{z}$ directions for $2 \mathrm{D}$ cases. The left side of equation 4 in the frequency domain can be expressed as

$$
\left(\mathrm{e}^{i \omega \Delta t}-2+\mathrm{e}^{-i \omega \Delta t}\right) \widetilde{Q}=[2 \cos (\omega \Delta t)-2] \widetilde{Q}=-4 \sin ^{2}(\omega \Delta t / 2) \widetilde{Q}
$$


where $\widetilde{Q}=F^{+}[Q(t)]$, and $F^{+}$represents the forward Fourier transform. The coefficient range of $\widetilde{Q}$ should be in $[-4,0]$. The matrix $A$ is a semi-negative definite matrix, like the updated matrix in FD, only has nonpositive real eigenvalues (Gaffar and Jiao, 2014; Li et al., 2014; Gao et al., 2018).

It has been demonstrated that the over-sampling nature of the spatial grids in the time-domain FD method, which is directly related to the construction of the matrix $A$ in the FD framework, provides the theoretical justification for extending the CFL limit (Li, 2014; Gao et al., 2018). And a time step $\Delta t>\Delta t_{\mathrm{CFL}}$ makes some eigenvalues of the matrix $A$ outside of $[-4,0]$, which leads to the unstable phenomena. Fortunately, $\mathrm{Li}$ (2014) proposes that an enforcement process may be performed by perturbing the unstable eigenvalues to ensure the stability, by retaining the stable eigenvalues without significantly affecting the accuracy. And this method is called the eigenvalue perturbation ( $\mathrm{Li}, 2014)$. All of these features have only direct relationships with the iterative matrix $A$ no matter it comes from the FD, the SEM or the other numerical wave propagation methods, so the iterative matrix $A$ constructed by the SEM should also be able to be perturbed. As a preprocessing step, the eigenvalue perturbation of the iterative matrix $A$ consists of the following three steps:

(1) compute the eigenvalue decomposition of the iterative matrix $A=\mathbf{W} \Lambda \mathbf{W}^{-1}$, where the matrix $\mathbf{W}$ includes all the eigenvectors, $\Lambda=\operatorname{diag}\left(\lambda_{1}, \lambda_{2}, \cdots, \lambda_{m}\right)$ is a diagonal matrix containing all the eigenvalues of the matrix $A$.

(2) perturb the unstable eigenvalues $\lambda_{i}<-4$ in $\Lambda$ to $\hat{\lambda}_{i}=-4$ to construct a new diagonal matrix $\hat{\Lambda}$ in order that the stability of equation 4 is remained.

(3) reconstruct a new updated iterative matrix $\hat{A}$ by the new perturbed eigenvalues. $\hat{A}=\mathbf{W} \hat{\Lambda} \mathbf{W}^{-1}$. The first prepossessing step can be time-consuming especially for large models (Gao et al., 2019). Nevertheless, some local eigenvalues approximation method can be further used to find the relevant eigenvalues to the poor CFL conditions (Yan and Jiao, 2017; Cottereau and Sevilla, 2018) for a reduced numerical cost. Moreover, this eigenvalue decomposition only needs to be performed only once throughout the whole workflow for a given model and a given spatial grid. After the eigenvalue perturbation, equation 4 can be rewritten as

$$
Q^{n+1}-2 Q^{n}+Q^{n-1}=\hat{A} Q^{n}+\Delta t^{2} M^{-1} F^{n}
$$


where $\hat{A}$ is the new perturbed updated matrix, and the size of the full $\hat{A}$ is the same as the sparse $A$ matrix. The second-order time derivative in the wave equation is typically computed by using the FD approximation. Koene et al. (2018) mentions that the FTDT and ITDT methods could also be used in SEM to remove the time-dispersion error for the second-order accurate Newmark scheme (Newmark, 1959). Here the second-order accurate Newmark scheme is used to update the $Q^{n}, \dot{Q}^{n}$ and $\ddot{Q}^{n}$ to the $Q^{n+1}, \dot{Q}^{n+1}$ and $\ddot{Q}^{n+1}$ as follows:

$$
\left\{\begin{array}{l}
Q^{n+1}=Q^{n}+\Delta t \dot{Q}^{n}+0.5 \Delta t^{2} \ddot{Q}^{n} \\
\dot{Q}^{n+1}=\dot{Q}^{n}+0.5 \Delta t\left[\ddot{Q}^{n}+\ddot{Q}^{n+1}\right] \\
\ddot{Q}^{n+1}=\frac{1}{\Delta t^{2}} \hat{A} Q^{n+1}+M^{-1} F^{n+1}
\end{array}\right.
$$

Note that the temporal central difference format and the above Newmark format are equivalent. From the Newmark scheme, it is known that

$$
Q^{n}=Q^{n-1}+\Delta t \dot{Q}^{n-1}+0.5 \Delta t^{2} \ddot{Q}^{n-1}
$$

Then we substract the first equation of equations 7 and equation 8 , after replacing $\left(\dot{Q}^{n}-\dot{Q}^{n-1}\right)$ by the second equation of equations 7 , their equivalence could be obtained as follows:

$$
\left\{\begin{array}{l}
Q^{n+1}-2 Q^{n}+Q^{n-1} \\
=\Delta t\left(\dot{Q}^{n}-\dot{Q}^{n-1}\right)+0.5 \Delta t^{2}\left(\ddot{Q}^{n}-\ddot{Q}^{n-1}\right) \\
=0.5 \Delta t^{2}\left(\ddot{Q}^{n}+\ddot{Q}^{n-1}\right)+0.5 \Delta t^{2}\left(\ddot{Q}^{n}-\ddot{Q}^{n-1}\right)=0.5 \Delta t^{2} \ddot{Q}^{n}
\end{array}\right.
$$

Thus, the corresponding FTDT and ITDT methods could be used in the above Newmark scheme to remove the time-dispersion error, which are derived from the temporal centre difference format.

It's also worth noting that, in the computation of the classical SEM, the global stiffness matrix $K$ is not usually assembled, and the product vector $K Q$, as a whole part, is calculated element by element based on the tensor product (Komatitsch and Vilotte, 1998). However, here we need assemble the global stiffness matrix $K$ to construct the updated matrix $A$, and then obtain the new updated matrix $\hat{A}$ through eigenvalue perturbation. 


\section{Eliminating the time-dispersion error from a time step beyond the CFL stability limit}

As the theoretical analyses illustrated above, now we could adopt a time step $\Delta t>\Delta t_{\mathrm{CFL}}$ to solve the equation 7 after applying the eigenvalue perturbation. However, it will result in an extremely large time-dispersion error due to the $\mathcal{O}\left(\Delta t^{2}\right)$ error accumulation for the second-order Newmark time scheme. Fortunately, we could eliminate the time-dispersion error by the combination of the FTDT and ITDT methods (Wang and Xu, 2015; Koene et al., 2018; Gao et al., 2018). A simplified introduction of the FTDT and ITDT methods is given below.

After the second-order time discretization at the left side of the equation 4, the relation between the original $\omega_{0}$ and time-dispersed $\omega$ frequencies is

$$
\text { 2nd-order : }\left\{\begin{array}{l}
-\omega_{0}^{2} \tilde{q} \approx \frac{\left(\mathrm{e}^{i \omega \Delta t}-2+\mathrm{e}^{-i \omega \Delta t}\right)}{\Delta t^{2}} \tilde{q} \\
\omega_{0} \approx \frac{2}{\Delta t} \sin \left(\frac{\omega \Delta t}{2}\right)
\end{array}\right.
$$

where $\widetilde{q}=F^{+}[q(t)]$. We thus could map the correct and dispersed phase rotations of a recording by adding dispersion in a forward Fourier transform to a trace $q(t)$ :

$$
\hat{q}(t)=\mathcal{F}^{-1}\left[\int_{-\infty}^{\infty} q(t) \mathrm{e}^{-i 2 \sin \left(\frac{\omega \Delta t}{2}\right) \frac{t}{\Delta t}} d t\right]
$$

We refer to this operation as the FTDT method. On the contrary, we could map a temporally dispersed signal back to the correct phase in a similar way as the FTDT method:

$$
q(t)=\mathcal{F}^{-1}\left[\int_{-\infty}^{\infty} \hat{q}(t) \mathrm{e}^{-i 2 \sin ^{-1}\left(\frac{\omega_{0} \Delta t}{2}\right) \frac{t}{\Delta t}} d t\right]
$$

And we refer to this operation as the ITDT method. For the sake of completeness, here we summarize the workflow about how to eliminate the time-dispersion error from a time step $\Delta t>\Delta t_{\mathrm{CFL}}$ by the combination of the FTDT method, the eigenvalue perturbation, and the ITDT method in the SEM framework as follows:

(1) Apply the FTDT method to the source time function. This preprocessing of the source ensures that we impose a wavelet with an identical amount of time-dispersion error as that accumulating in the wavefields (Koene et al., 2018). 
(2) Perform the acoustic wave numerical simulation with the new updated matrix $\hat{A}$ and a time step $\Delta t>\Delta t_{\mathrm{CFL}}$ by the SEM, and record the desired traces.

(3) Apply the ITDT method to the recorded traces. This step can recover the original waveforms without the time-dispersion error.

\section{NUMERICAL EXPERIMENTS}

To further numerically validate the combined method, numerical experiments in the $2 \mathrm{D}$ homogeneous in the 2D homogeneous, moderate and strong heterogeneous models are followed.

\section{D homogeneous model case}

Firstly, we carry out numerical experiments in a 2D homogeneous square model with the size $2000 \mathrm{~m} \times 2000 \mathrm{~m}$ by the SEM. The wave velocity is constant with $V=4000 \mathrm{~m} / \mathrm{s}$. The only receiver is chosen at $(0 \mathrm{~m}, 2000 \mathrm{~m})$. And the source, located at the center of the model, is a $20 \mathrm{~Hz}$ Ricker wavelet. In terms of the memory of the updated matrix $A$, we adopt only one element with 121 GLL points in $\mathrm{x}$ and $\mathrm{z}$ orthogonal directions for the spatial meshing, which leads to almost no spatial-dispersion error based on the empirical value of points per minimum wavelength $G \approx 3.2$ (Seriani and Priolo, 1994; Capdeville et al., 2010). The corresponding maximum time step available for the second-order temporal discretization is $\Delta t_{\mathrm{CFL}} \approx 0.076 \mathrm{~ms}$ (Courant constant $\approx 0.6$ ). The spatial interval $\Delta x_{\min }$ is too small to be adapted in real numerical simulations. After using the combined method, we verify five larger time steps: $\Delta t=1,3,5,7$ and $9 \mathrm{~ms}$, which are all tens of times larger than $\Delta t_{\mathrm{CFL}}$. A dense enough meshing containing $40 \times 40$ elements with 11 GLL points $(G \approx 10.7)$ and a small enough time step $(\Delta t=0.1 \mathrm{~ms})$ are used to obtain the reference waveforms and snapshots without spatial and time-dispersion errors. And they will be used to benchmark the numerical accuracy of the waveforms and snapshots calculated by the five larger time steps.

Figure 1 displays four snapshots at 1470 ms simulated by SEM with four different time steps, and Figure 2 shows the numerical waveforms with five time steps and the associated reference waveform. Figures $1 \mathbf{a}$ and $2 \mathbf{a}$ show that, as the time step $\Delta t$ increases the time-dispersion error becomes more and more obvious and serious in the snapshots and waveforms. After applying the 
ITDT method, the time-dispersion error is clearly eliminated as shown by the snapshots in Figure $1 \mathbf{b}$ and in the waveforms by Figure $2 \mathbf{b}$. The four snapshots in Figure $1 \mathbf{b}$ are almost the same, and the waveforms from $1 \mathrm{~ms}$ to $7 \mathrm{~ms}$ in Figure $2 \mathbf{b}$ are highly overlapped with the referenced waveforms. All of the above snapshots and waveforms demonstrate that, in the SEM framework, eigenvalue perturbation can extend the CFL stability limit and the resultant time-dispersion error can be further eliminated by the FTDT and ITDT methods. That means in the 2D homogeneous model we could use a time step beyond the $\Delta t_{\mathrm{CFL}}$, and the resultant waveforms have no time-dispersion error, which is in agreement with the FD case in Gao et al. (2018). Even though the time-dispersion error using $\Delta t=7 \mathrm{~ms}$ is not very obvious after ITDT method, some oscillations suddenly arise near the source location in Figure $1 \mathbf{b} 4$ and in Figure $2 \mathbf{b}$ for the $\Delta t=7 \mathrm{~ms}$ case at about $2.95 \mathrm{~s}$. And these phenomena become uncontrollable and unusable when the $\Delta t$ is chosen towards or above the Nyquist sampling time step $\Delta t_{\mathrm{Nyq}}=1 /\left(2 f_{\max }\right)$. For the Ricker wavelet we used, the maximum frequency is about $70 \mathrm{~Hz}$, and the time step should be smaller than $7.1 \mathrm{~ms}$ depending on the Nyquist limit $\Delta t<\Delta t_{\mathrm{Nyq}}$. The waveform from $\Delta t=9 \mathrm{~ms}$ in Figure $2 \mathbf{b}$ is not accurate any more especially for the high frequency components, due to the insufficient sampling of source frequency spectrum. Thus, the time step cannot be infinitely large and still constrained by the Nyquist sampling theorem.

\section{D heterogenous cosine model case}

Furthermore, we validate the combined method in a 2D smooth cosine heterogeneous model, which is a typical representation of the homogenized model. Its oscillating property with space needs to be accurately integrated, and a larger spatial sampling $G$ than that in homogeneous models (Capdeville et al., 2010) needs to be adapted. Our cosine model has the size of $2000 \mathrm{~m} \times 2000 \mathrm{~m}$, and its velocity range $V$ is from 3600 to $4400 \mathrm{~m} / \mathrm{s}$, which is a moderate variation of the velocity. The source time function is the same as the homogeneous case. The only receiver is located at $(0 \mathrm{~m}, 2000 \mathrm{~m})$. The associated spatial meshing includes $8 \times 8$ elements with 15 GLL points as shown in Figure $3 \mathbf{a}$, which leads to almost no spatial-dispersion error owing to the good enough $G \approx 3.2$ (Seriani and Priolo, 1994; Capdeville et al., 2010). The associated maximum time step for the second-order Newmark temporal discretization is $\Delta t_{\mathrm{CFL}} \approx 0.6 \mathrm{~ms}$ (courant constant $\approx 0.608$ ). Similar to the homogeneous case, we verify five larger time steps: $\Delta t=1,3,5,7$ and $9 \mathrm{~ms}$. And the reference 
waveform is calculated by the same meshing and a fine time step $\Delta t=0.05 \mathrm{~ms}$ almost without the time-dispersion error.

After the whole procedure of the combined method, the residuals between the numerical and reference waveforms in Figure $3 \mathbf{b}$ are unapparent even for $\Delta t=7 \mathrm{~ms}$ (only with a little waveform oscillation), which implies that the obtained numerical waveforms have almost no time-dispersion error. The waveform from $\Delta t=9 \mathrm{~ms}$ in Figure $3 \mathbf{b}$ is unusable due to the insufficient sampling of source frequency spectrum. This numerical experiment validates that through the combined method, in the SEM framework, we can adopt a time step, far beyond the CFL stability limit and below the Nyquist sampling theorem, to obtain the resultant waveforms without time-dispersion error in the general homogenized models with moderate velocity variation as well.

\section{D modified Marmousi model case}

In the last numerical experiment, we validate the combined method in a 2D modified Marmousi model with a very long time simulation (about 1000 periods). Its spatially varying property also needs to be accurately integrated, and a larger spatial sampling $G$ than that in homogeneous models needs to be adapted. Our model has the size of $2000 \mathrm{~m} \times 2000 \mathrm{~m}$, and its velocity range $V$ is from 1500 to $5443 \mathrm{~m} / \mathrm{s}$, which is a strong variation of the velocity. The source, located at the center of the model, is a $15 \mathrm{~Hz}$ Ricker wavelet. The only receiver is located at $(0 \mathrm{~m}, 0 \mathrm{~m})$. The associated spatial meshing includes $10 \times 5$ elements with 21 GLL points as shown in Figure 4a, which leads to almost no spatial-dispersion error owing to the good precise $G \approx 2.9$ for the very high order SEM (Seriani and Priolo, 1994; Capdeville et al., 2010). The associated maximum time step for the second-order Newmark temporal discretization is $\Delta t_{\mathrm{CFL}} \approx 0.195 \mathrm{~ms}$ (Courant constant $\approx 0.61$ ). Similarly, we verify five larger time steps: $\Delta t=3,5,7,9$ and $11 \mathrm{~ms}$. They are slightly larger than the above two cases, because the used maximum frequency $52.5 \mathrm{~Hz}$ is smaller than the $70 \mathrm{~Hz}$ in the above cases, we could have a larger Nyquist sampling time step about $9.5 \mathrm{~ms}$. And the reference waveform is calculated by the same meshing and a small enough time step $\Delta t=0.05 \mathrm{~ms}$ almostly without time-dispersion error. After the calculation of using the combined method, the residuals between the numerical and reference waveforms in Figure $4 \mathbf{b}$ are unapparent even for $\Delta t=9 \mathrm{~ms}$ (only with a little waveform oscillation), which implies that the obtained numerical waveforms have 
almostly no time-dispersion error.

In summary, for the homogeneous, moderate and strong heterogeneous models, we use time steps beyond the CFL limit even towards the Nyquist limit, and the resultant waveforms almostly have no time-dispersion error by the combined methods. For the time step above the Nyquist limit, the waveforms from $\Delta t=9 \mathrm{~ms}$ in Figures $2 \mathbf{b}$ and $3 \mathbf{b}$ and the waveform from $\Delta t=11 \mathrm{~ms}$ in Figure $4 \mathbf{b}$ are not accurate any more especially for the high-frequency components, due to the insufficient sampling of source frequency spectrum.

\section{DISCUSSION}

As one of the methods to eliminate or extend the CFL stability limit of explicit scheme at present, the spatial filtering method is a more basic approach to understand the exact meaning of the CFL stability limit and how to extend this limit. However, the eigenvalue perturbation method performs better than the spatial filtering method for its feasibility for strongly inhomogeneous media.

It is well known that the GLL points cluster towards the edge of the element for very high degree SEM, which seriously restricts its application especially for the model with fine structures. And the small available time steps of using the very high degree SEM for the homogenized model still constraint its real application. Based on the combined method, the time step is not limited by the minimum spatial GLL interval $\Delta x_{\min }$ anymore, which is a huge advantage because the very high-degree SEM with a low G can be used to perform simulation without the spatial- and timedispersion errors. As the homogenous numerical experiment shows, the very high-degree SEM can adopt tens of times larger than $\Delta t_{\mathrm{CFL}}$ to perform simulation owing to its extremely small space. However, for FD method due to its general uniform spatial grid, the available time steps are only several times of the $\Delta t_{\mathrm{CFL}}$. It seems that the resultant waveform calculated by the time steps near the Nyquist sampling in SEM is much stabler than the corresponding waveform in the FD, which can be observed between the Figure $3 \mathbf{b}$ in Gao et al. (2019) and the Figure $2 \mathbf{b}$ in this paper.

It's also worth noting that the eigenvalue perturbation of the iterative matrix $A$ is a pre-processing procedure, and only needs to be calculated once throughout the whole workflow for a given model and a given spatial grid. This will greatly reduce the calculation time for some technical methods, 
such as the full waveform inversion and migration who require numerical simulations of multiple sources for the same model, which shows a potential application prospect. Although the combined method with the SEM is very promising, until now it is still very difficult to be applied in large models. The new updated iterative matrix $\hat{A}$ is full, so a lot of memory and large computational sources are needed. For a 2D model meshed by $20 \times 20$ spectral elements with 11 GLL points in each orthogonal direction, the memory cost of matrix $\hat{A}$ is about $6.0 \mathrm{~GB}$. And the calculation of eigenvalue of matrix $A$ increases as exponential as the number of points (Gao et al., 2018), which greatly limits the application of this method in practice. And in order to build the updated iterative matrix $\hat{A}$, we need the global stiffness matrix $K$. However, for the classic SEM, the global stiffness matrix $K$ is not usually built, and the product vector $K Q$ is calculated element by element based on the tensor product as a whole part. For example, in the calculation of our Marmousi model case, the size of the matrix $A$ is $20301 \times 20301$, which takes 4619 s for the eigenvalue related calculation. While in the following SEM long time simulation (about 1000 periods) with the full stiffness matrix $\hat{A}$, it takes $3058 \mathrm{~s}$. And the calculation of the reference waveform with fine time step $\Delta t=0.05 \mathrm{~s}$ takes $1436 \mathrm{~s}$, which is based on the traditional spectral-element method in the element by element way also shown in Table 1.

Next, we compare the computational complexity analysis of the acoustic FWI by SEM numerical simulation and eigenvalue calculation. For the acoustic FWI in the $d$ dimensions with $n s$ sources, $n r$ receivers, and $n t$ time steps, regarding the number of elements $n e^{d}$ with the element degree $N$, the corresponding computational complexity is $n t \times(n s+n r) \times n e^{d} \times N^{d+1}$. Note that $N^{d+1}$ is usually where the main optimization effort is put into, following matrix-matrix multiplication strategy (Deville et al., 2002). The conservative computational complexity of the eigenvalue calcualation is about $O\left(n^{3}\right)$, where $n$ is the number of degrees of freedom. In previous same model, the associated computational complexity is $O\left(\left((n e \times N+1)^{d}\right)^{3}\right) \approx O\left((n e \times N)^{3 d}\right)$, for example, by the QR algorithm (Parlett, 2000). And the ratio between SEM and eigenvalue calculation is about $O\left(\frac{n t \times(n s+n r)}{n e^{2 d} \times N^{2 d-1}}\right)$. They are both in the usual numerical linear algebra statistics where the basic operations $(+,-, *, /)$ are performed approximately in current machine arithmetic and cost $\mathrm{O}(1)$ each. Unless the computational complexity of solving eigenvalues increases significantly, obtaining all the eigenvalues and applying the eigenvalue perturbation for 3D large-scale models will remain 
impossible. So to avoid globally calculating the stiffness matrix $K$ and the eigenvalues of large matrices for real applications, some local eigenvalues approximation method can be further used to eliminate the relevant eigenvalues that destroy the CFL conditions to make use of the larger time step $\Delta t>\Delta t_{\mathrm{CFL}}$ (Yan and Jiao, 2017; Cottereau and Sevilla, 2018). In the global seismology, the eigenvalue calculation can be parallelized by the angular orders (wave number) for the spherically symmetric earth models as used in Capdeville et al. (2003) to calculate the limited normal modes (Takeuchi and Saito, 1972). For the more general cases, the MPI and OpenMP could be used for the parallelization computation of finding some eigenvalues, such as the FEAST package (Polizzi, 2009): http://www.ecs.umass.edu/ polizzi/feast/. After obtaining the new iterative matrix by eigenvalue perturbation, one could store it for all subsequent forward simulations in the model. And another very important content about how to further implement the combined method in the numerical modeling with absorbing boundary conditions is also an issue to be addressed in the future.

\section{CONCLUSION}

In the explicit time-domain SEM framework for solving the 2D acoustic wave equation, based on the verifications of theoretical analyses and numerical experiments, we succeed in combining the FTDT method, the eigenvalue perturbation, and ITDT method to use time steps beyond the CFL limit even towards the Nyquist limit, and the resultant waveforms exhibit no time-dispersion error. It lays an initial foundation that we probably could draw lessons from all the efficient eigenvalue calculation methods to perform the numerical wave simulation by the SEM with a very large time step $\Delta t>\Delta t_{\mathrm{CFL}}$ in the complex models.

\section{ACKNOWLEDGEMENTS}

This study is supported by the National Key R\&D Program of China (Grant No. 2017YFC0601206), NSFC (grant no. 41888101, 42004045, 41625016, and 41774112), the China Scholarship Council (File No. 201804910289) and the French National Research Agency (ANR-16-CE31-0022-01, 2016-2021). We thank the editor-in-chief Jeffrey Shragge, the assistant editors Joakim Blanch and 
Stig Hestholm, and five anonymous reviewers, for their comments which greatly helped to improve the manuscript. Chao Lyu also sincerely thank Yingjie Gao and Yan Jin for plentiful discussions on the CFL's removal.

\section{REFERENCES}

Capdeville, Y., , J. P. Vilotte, and J. P. Montagner, 2003, Coupling the spectral element method with a modal solution for elastic wave propagation in global earth models: Geophysical Journal International, 152, 34-67.

Capdeville, Y., L. Guillot, and J. Marigo, 2010, 2-d non-periodic homogenization to upscale elastic media for p-sv waves: Geophysical Journal International, 182, 903-922.

Capdeville, Y., and L. Métivier, 2018, Elastic full waveform inversion based on the homogenization method: theoretical framework and 2-d numerical illustrations: Geophysical Journal International, 213, 1093-1112.

Chaljub, E., D. Komatitsch, Y. Capdeville, J.-P. Vilotte, B. Valette, and G. Festa, 2007, Spectral element analysis in seismology: Advances in Wave Propagation in Heterogeneous Media, 48, $365-419$.

Chang, C., and C. D. Sarris, 2011, A spatial filter-enabled high-resolution subgridding scheme for stable fdtd modeling of multiscale geometries: 2011 IEEE MTT-S International Microwave Symposium, 1-4.

Cottereau, R., and R. Sevilla, 2018, Stability of an explicit high-order spectral element method for acoustics in heterogeneous media based on local element stability criteria: International Journal for Numerical Methods in Engineering, 116, 223-245.

Cupillard, P., and Y. Capdeville, 2018, Non-periodic homogenization of 3-d elastic media for the seismic wave equation: Geophysical Journal International, 213, 983-1001.

Deville, M. O., P. F. Fischer, and E. H. Mund, 2002, High-order methods for incompressible fluid flow: Cambridge University Press. Cambridge Monographs on Applied and Computational Mathematics.

Ecer, A., N. Gopalaswamy, H. U. Akay, and Y. P. Chien, 2000, Digital filtering techniques for parallel computation of explicit schemes: International Journal of Computational Fluid Dynamics, 
13, 211-222.

Etgen, J., 1994, Stability of explicit depth extrapolation through laterally-varying media: 64th Annual International Meeting, SEG, Expanded Abstracts.

Fichtner, A., B. L. N. Kennett, H. Igel, and H. Bunge, 2009, Full seismic waveform tomography for upper-mantle structure in the Australasian region using adjoint methods: Geophysical Journal International, 179, 1703-1725.

Fichtner, A., D.-P. van Herwaarden, M. Afanasiev, S. Simute, L. Krischer, Y. Çubuk Sabuncu, T. Taymaz, L. Colli, E. Saygin, A. Villaseñor, J. Trampert, P. Cupillard, H.-P. Bunge, and H. Igel, 2018, The collaborative seismic earth model: Generation 1: Geophysical Research Letters, 45, 4007-4016.

French, S. W., and B. Romanowicz, 2015, Broad plumes rooted at the base of the earth's mantle beneath major hotspots: Nature, 525, 95 .

Gaffar, M., and D. Jiao, 2014, An explicit and unconditionally stable fdtd method for electromagnetic analysis: IEEE Transactions on Microwave Theory and Techniques, 62, 2538-2550.

Gao, Y., J. Zhang, and Z. Yao, 2016, Third-order symplectic integration method with inverse time dispersion transform for long-term simulation: Journal of Computational Physics, 314, 436-449.

$\ldots$ _ 2019, Extending the stability limit of explicit scheme with spatial filtering for solving wave equations: Journal of Computational Physics, 397, 108853.

Gao, Y., J. Zhang, and Y. Zhenxing, 2018, Removing the stability limit of the explicit finitedifference scheme with eigenvalue perturbation: Geophysics, 83, no. 6, A93-A98.

He, Q., H. Gan, and D. Jiao, 2012, Explicit time-domain finite-element method stabilized for an arbitrarily large time step: IEEE Transactions on Antennas and Propagation, 60, 5240-5250.

Jin, Y., and D. Jiao, 2016, Explicit and unconditionally stable fdtd method without eigenvalue solutions: 2016 IEEE MTT-S International Microwave Symposium (IMS), 1-4.

Koene, E. F. M., J. O. A. Robertsson, F. Broggini, and F. Andersson, 2018, Eliminating time dispersion from seismic wave modeling: Geophysical Journal International, 213, 169-180.

Komatitsch, D., and J.-P. Vilotte, 1998, The spectral element method : an effective tool to silmulate the seismic response of $2 \mathrm{~d}$ and $3 \mathrm{~d}$ geological structures: Bulletin of the seismologic Society of America, 88, 368-392.

Li, X., 2014, Model order reduction and stability enforcement of finite-difference time-domain 
equations beyond the cfl limit: Master dissertation, Univeristy of Toronto.

Li, X., C. D. Sarris, and P. Triverio, 2014, Overcoming the fdtd stability limit via model order reduction and eigenvalue perturbation: International Microwave Symposium (IMS2014), 1-3.

Lyu, C., Y. Capdeville, D. Al-Attar, and L. Zhao, 2021, Intrinsic non-uniqueness of the acoustic full waveform inverse problem: Geophysical Journal International.

Lyu, C., Y. Capdeville, and L. Zhao, 2020, Efficiency of the spectral element method with very high polynomial degree to solve the elastic wave equation: Geophysics, 85, no. 1, T33-T43.

Newmark, N., 1959, A method of computation for structural dynamics: Journal of the Engineering Mechanics Division, ASCE, 85.

Parlett, B. N., 2000, The qr algorithm: Computing in science \& engineering, 2, P.38-42.

Polizzi, E., 2009, Density-matrix-based algorithm for solving eigenvalue problems: Phys. Rev. B, 79, no. 11,115112 .

Sarris, C. D., 2011, Extending the stability limit of the fdtd method with spatial filtering: IEEE Microwave and Wireless Components Letters, 21, 176-178.

Seriani, G., and E. Priolo, 1994, Spectral element method for acoustic wave simulation in heterogeneous media: Finite Elements in Analysis and Design, 16, 337 - 348. (Special Issue Selection of Papers Presented at ICOSAHOM'92).

Stork, C., 2013, Eliminating nearly all dispersion error from fd modeling and $\mathrm{rtm}$ with minimal cost increase: 75th Annual International Conference and Exhibition EAGE, Extended Abstracts.

Takeuchi, H., and M. Saito, 1972, Seismic surface waves: Methods in computational Physics., 11, 217-295.

Tromp, J., 2020, Seismic wavefield imaging of earth's interior across scales: Nature Reviews Earth \& Environment, 1, 40-53.

Tromp, J., C. Tape, and Q. Liu, 2005, Seismic tomography, adjoint methods, time reversal and banana-doughnut kernels: Geophysical Journal International, 160, 195-216.

Wang, M., and S. Xu, 2015, Finite-difference time dispersion transforms for wave propagation: Geophysics, 80, no. 6, WD19-WD25.

Yan, J., and D. Jiao, 2017, Fast explicit and unconditionally stable fdtd method for electromagnetic analysis: IEEE Transactions on Microwave Theory and Techniques, 65, 2698-2710.

Zhu, H., E. Bozdağ, D. Peter, and J. Tromp, 2012, Structure of the european upper mantle revealed 
by adjoint tomography: Nature Geoscience, 5, 493-498. 
Table 1:Comparison of calculated time for the Marmousi model case. The computations are finished in the 2020 MacBook Pro16 with 2.4 GHz core and 64G memory in Matlab 2020b. 


\section{Figure Captions}

- Figure 1: Snapshots obtained by four different time steps at $1470 \mathrm{~ms}$. (a) (a1-a4) are obtained by time steps $1,3,5$, and $7 \mathrm{~ms}$ with the FTDT method and eigenvalue perturbation respectively. (b) (b1-b4) are the corresponding snapshots in (a) after applying the ITDT method.

- Figure 2: Waveforms calculated by five different time steps. (a) are the waveforms obtained by time steps from $1 \mathrm{~ms}$ to $9 \mathrm{~ms}$ after using the FTDT method and eigenvalue perturbation. The five waveforms in black solid lines from bottom to top are calculated by time steps from $1 \mathrm{~ms}$ to $9 \mathrm{~ms}$ respectively, and the each red dashed line displays the reference waveform calculated by a very small $\Delta t$. (b) are the corresponding black waveforms in (a) after applying the ITDT method, shown with black solid lines, and the red dashed line is the reference waveform.

- Figure 3: Configuration of the smooth 2D cosine heterogeneous model and the waveforms calculated by five different time steps with a relatively long time simulation. (a) The 2D cosine model. The gray solid lines display the spatial meshing by the $8 \times 8$ spectral elements. The red star presents the location of the source and the blue triangle presents the receiver. The velocity $V(\mathbf{x})=V_{0} f(\mathbf{x})$, where $V_{0}$ is the constant velocity used in the homogeneous case. And the heterogeneous mechanical properties have been chosen with the form $f(\mathbf{x})=1+$ $a\left(\cos \left(\frac{2 \pi}{T_{h}}\left(\mathbf{k}_{a} \cdot \mathbf{x}\right)+\cos \left(\frac{2 \pi}{\lambda_{h}}\left(\mathbf{k}_{b} \cdot \mathbf{x}\right)\right)\right)\right.$, where $\mathbf{k}_{a}=\left(\cos \left(t_{1}\right), \sin \left(t_{1}\right)\right), \mathbf{k}_{b}=\left(\cos \left(t_{2}\right), \sin \left(t_{2}\right)\right)$. The amplitude $a$ is adapted 0.5 and the two crossed directions are $t_{1}=\frac{\pi}{4}, t_{2}=\frac{3 \pi}{4}$. The model spatial wavelength $\lambda_{h}=50 \mathrm{~m}$. (b) Waveforms calculated by five different time steps $\Delta t$, from $1 \mathrm{~ms}$ to $9 \mathrm{~ms}$. The corresponding black waveforms shown with black solid lines are calculated by applying the combination of the FTDT method, the eigenvalue perturbation and the ITDT method, and the red dashed line is the reference waveform calculated by a fine time step.

- Figure 4: Configuration of the 2D smooth modified Marmousi model and the waveforms calculated by five different time steps with a relatively long time simulation. (a) The modified Marmousi model. The gray solid lines display the spatial meshing by the $10 \times 5$ spectral elements. The red star presents the location of the source and the blue triangle presents the receiver. The velocity range is from 1500 to $5500 \mathrm{~m} / \mathrm{s}$. (b) Waveforms calculated by five 
different time steps $\Delta t$, from $3 \mathrm{~ms}$ to $11 \mathrm{~ms}$. The corresponding black waveforms shown with black solid lines are calculated by applying the combination of the FTDT method, the eigenvalue perturbation and the ITDT method, and the red dashed line is the reference waveform computed by a fine time step. 
Table 1: Comparison of calculated time for the Marmousi model case. The computations are finished in the 2020 MacBook Pro16 with $2.4 \mathrm{GHz}$ core and 64G memory in Matlab 2020b.

\begin{tabular}{|c|c|c|}
\hline Conventional SEM with $\Delta t=0.05 \mathrm{~ms}$ & Eigenvalue perturbation & SEM with full K with $\Delta t=11 \mathrm{~ms}$ \\
\hline $1436 \mathrm{~s}$ & $4619 \mathrm{~s}$ & $3058 \mathrm{~s}$ \\
\hline
\end{tabular}




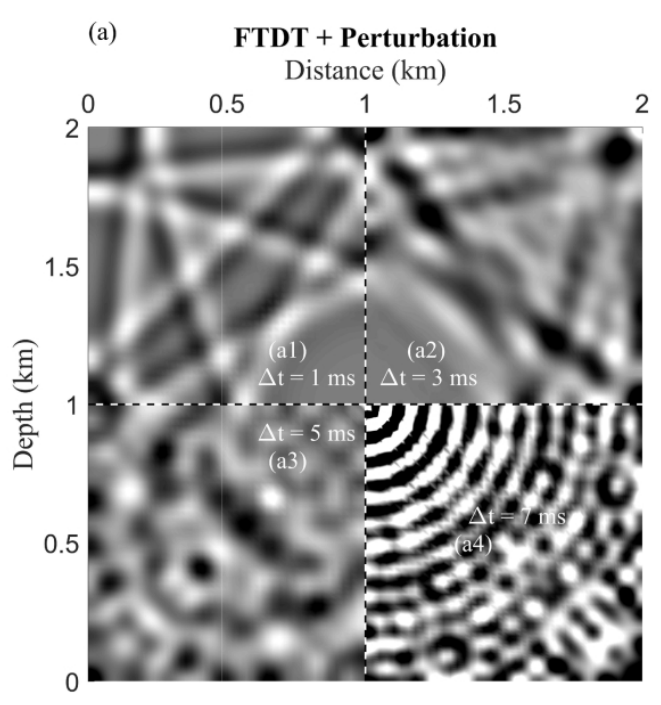

(b) FTDT + Perturbation + ITDT

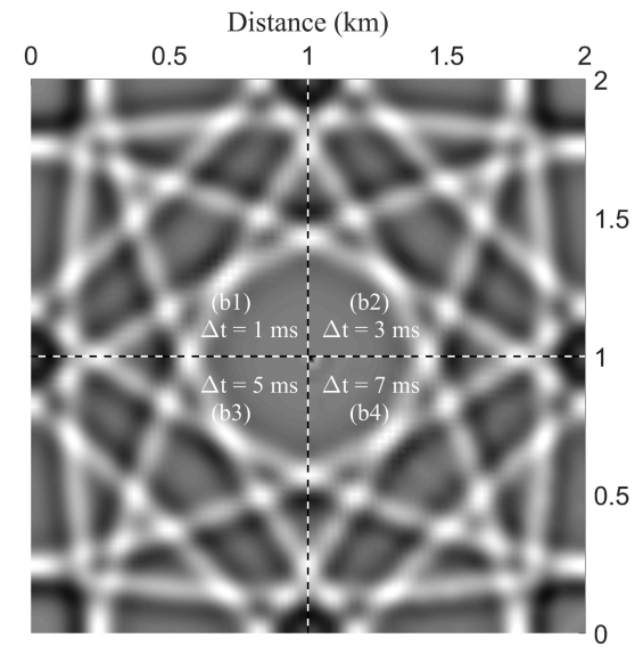

Figure1: Snapshots obtained by four different time steps at $1470 \mathrm{~ms}$. (a) (a1-a4) are obtained by time steps 1, 3, 5, and 7 ms with the FTDT method and eigenvalue perturbation respectively. (b) (b1-b4) are the corresponding snapshots in (a) after applying the ITDT method.

\section{$249 \times 132 \mathrm{~mm}(300 \times 300$ DPI)}


(a)

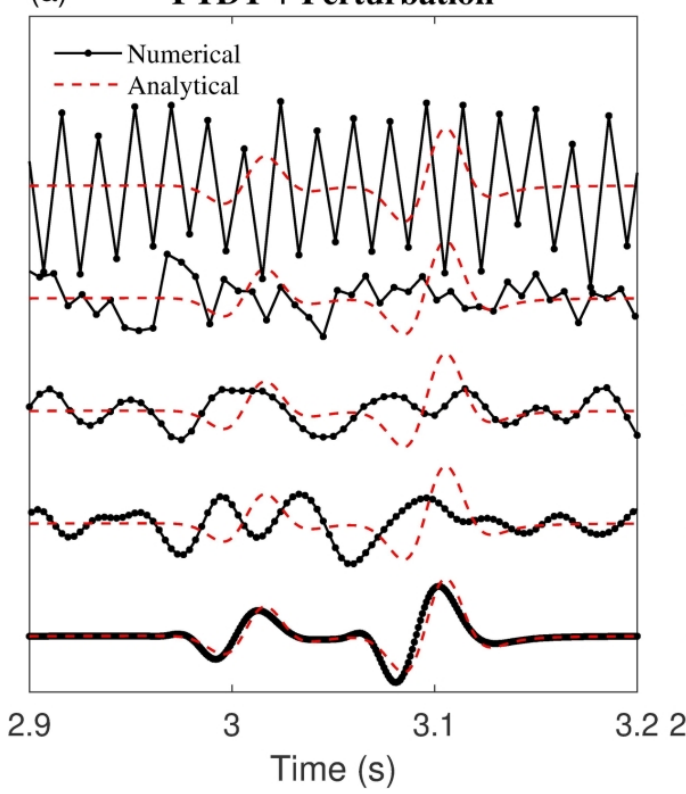

(b) FTDT + Perturbation + ITDT

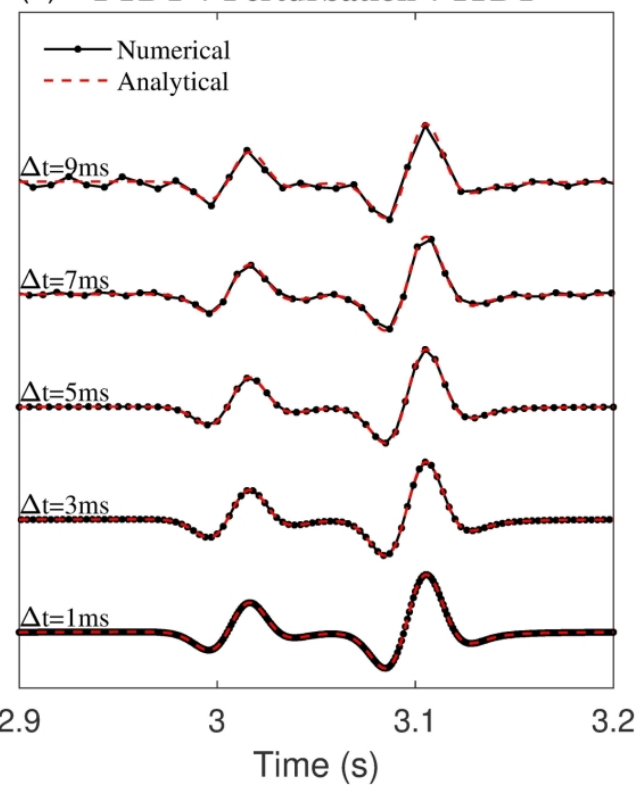

Figure2: Waveforms calculated by five different time steps. (a) are the waveforms obtained by time steps from $1 \mathrm{~ms}$ to $9 \mathrm{~ms}$ after using the FTDT method and eigenvalue perturbation. The five waveforms in black solid lines from bottom to top are calculated by time steps from $1 \mathrm{~ms}$ to $9 \mathrm{~ms}$ respectively, and each red dashed line displays the reference waveform calculated by a very small $\Delta \mathrm{t}$. (b) are the corresponding black waveforms in (a) after applying the ITDT method, shown with black solid lines, and the red dashed line is the reference waveform.

$203 \times 127 \mathrm{~mm}(300 \times 300 \mathrm{DPI})$ 


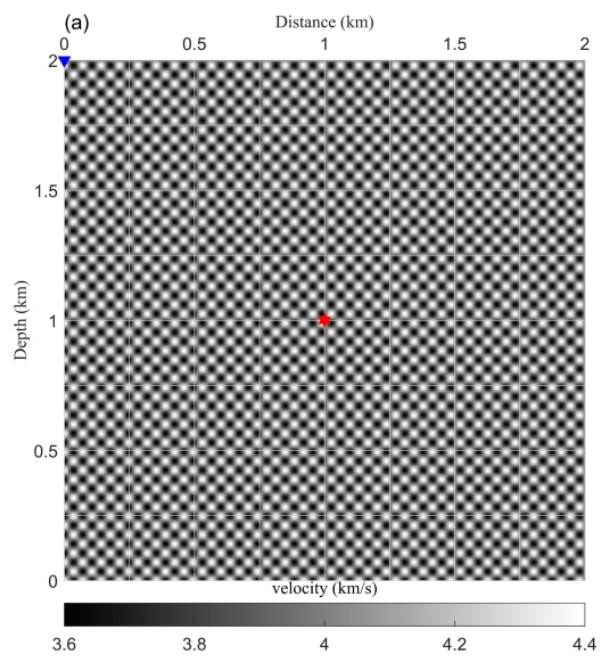

(b)

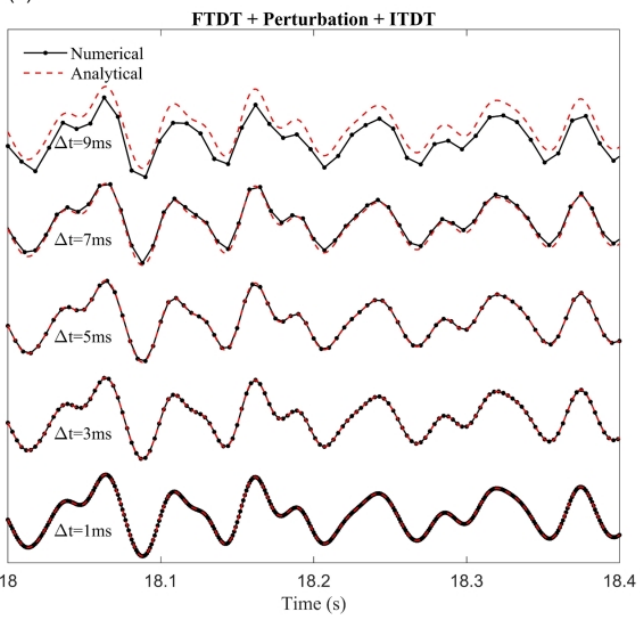

Figure3: Configuration of the smooth 2D cosine heterogeneous model and the waveforms calculated by five different time steps with a relatively long time simulation. (a) The 2D cosine model. The gray solid lines display the spatial meshing by the $8 \times 8$ spectral elements. The red star presents the location of the source and the blue triangle presents the receiver. The velocity $V(x)=V_{0} f(x)$, where $V_{0}$ is the constant velocity used in the homogeneous case. And the heterogeneous mechanical properties have been chosen with the form $f(x)=1+a\left(\cos \left(2 \pi / T_{h}(k a \cdot x)+\cos \left(2 \pi / \lambda_{h}(k b \cdot x)\right)\right)\right.$, where $k a=\left(\cos \left(t_{1}\right), \sin \left(t_{1}\right)\right), k b=\left(\cos \left(t_{2}\right)\right.$, $\left.\sin \left(t_{2}\right)\right)$. The amplitude $a$ is adapted 0.5 and the two crossed directions are $t 1=n / 4, t 2=3 / 4 n$. The model spatial wavelength $\lambda_{h}=50 \mathrm{~m}$. (b) Waveforms calculated by five different time steps $\Delta \mathrm{t}$, from $1 \mathrm{~ms}$ to $9 \mathrm{~ms}$. The corresponding black waveforms shown with black solid lines are calculated by applying the combination of the FTDT method, the eigenvalue perturbation and the ITDT method, and the red dashed line is the reference waveform calculated by a fine time step.

$317 \times 159 \mathrm{~mm}(300 \times 300 \mathrm{DPI})$ 
(b)

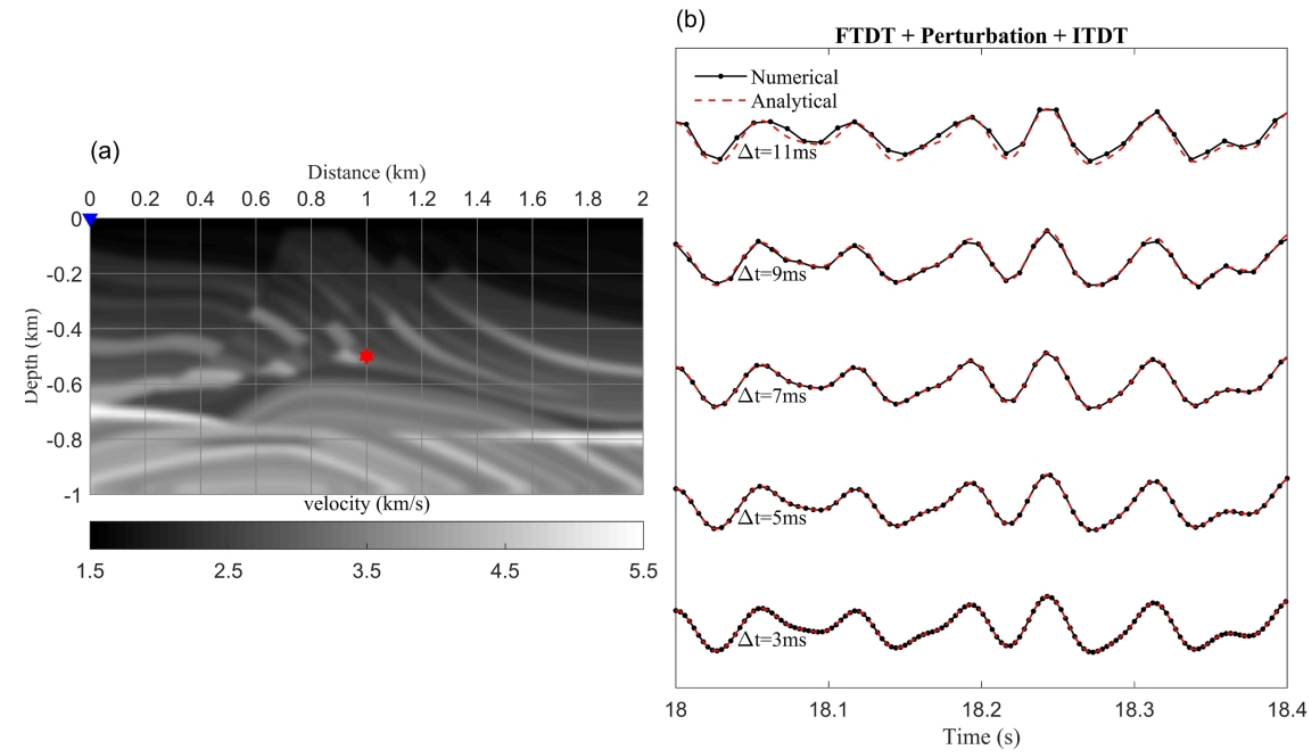

Figure4: Configuration of the 2D smooth modified Marmousi model and the waveforms calculated by five different time steps with a relatively long time simulation. (a) The modified Marmousi model. The gray solid lines display the spatial meshing by the $10 \times 5$ spectral elements. The red star presents the location of the source and the blue triangle presents the receiver. The velocity range is from 1500 to $5500 \mathrm{~m} / \mathrm{s}$. (b)

Waveforms calculated by five different time steps $\Delta t$, from $3 \mathrm{~ms}$ to $11 \mathrm{~ms}$. The corresponding black waveforms shown with black solid lines are calculated by applying the combination of the FTDT method, the eigenvalue perturbation and the ITDT method, and the red dashed line is the reference waveform computed by a fine time step.

$264 \times 159 \mathrm{~mm}(300 \times 300 \mathrm{DPI})$ 
DATA AND MATERIALS AVAILABILITY

Data associated with this research are available and can be obtained by contacting the corresponding author. 